\title{
Eye gaze perception in bipolar disorder: Self-referential bias but intact perceptual sensitivity
}

\author{
Beier Yao $^{1}$ (D) | Savanna A Mueller ${ }^{2}$ | Tyler B Grove ${ }^{2}$ | Merranda McLaughlin ${ }^{3}$ | \\ Katharine Thakkar ${ }^{1}$ | Vicki Ellingrod ${ }^{2,3,4}$ | Melvin G Mclnnis ${ }^{3}$ | Stephan F Taylor ${ }^{3}$ | \\ Patricia J Deldin ${ }^{2,3}$ | Ivy F Tso ${ }^{2,3}$
}

${ }^{1}$ Department of Psychology, Michigan State University, East Lansing, MI, USA

${ }^{2}$ Department of Psychology, University of Michigan, Ann Arbor, MI, USA

${ }^{3}$ Department of Psychiatry, University of Michigan, Ann Arbor, MI, USA

${ }^{4}$ College of Pharmacy, University of Michigan, Ann Arbor, MI, USA

\section{Correspondence}

Ivy F. Tso, Department of Psychiatry, University of Michigan, Rachel Upjohn Building, 4250 Plymouth Road, Ann Arbor, MI 48109, USA.

Email: ivytso@umich.edu

\section{Funding information}

National Institutes of Health, Grant/Award Number: 5KL2TR000434; National Institute of Mental Health, Grant/Award Number: 5K23MH108823, R21MH101676 and R01MH082784; Brain and Behavior Research Foundation, Grant/Award Number: NARSAD Young Investigator award; Heinz C. Prechter Bipolar Research Fund
Objectives: Deficits in social cognition predict poor functional outcome in severe mental illnesses such as schizophrenia and autism. However, research findings on social cognition in bipolar disorder (BD) are sparse and inconsistent. This study aimed to characterize a critical social cognitive process-eye gaze perception-and examine its functional correlates in BD to inform psychopathological mechanisms.

Methods: Thirty participants with BD, 37 healthy controls (HC), and 46 psychiatric controls with schizophrenia (SZ) completed an eye-contact perception task. They viewed faces with varying gaze directions, head orientations, and emotion, and made eye-contact judgments. Psychophysics methods were used to estimate perception thresholds and the slope of the perception curve, which were then compared between the groups and correlated with clinical and functional measures using Bayesian inference.

Results: Compared with HC, patients with BD over-perceived eye contact when gaze direction was ambiguous, and this self-referential bias was similar to that in SZ. Patients with BD had lower thresholds (i.e., needed weaker eye-contact signal to start perceiving gaze as self-directed) but a similar slope compared with HC. Regression analyses showed that steeper slope predicted better socio-emotional functioning in $\mathrm{HC}$ and SZ, but not in BD.

Conclusions: The psychopathology of social dysfunction was fundamentally different between BD and SZ in this modest sample. Eye gaze perception in BD was characterized by a self-referential bias but preserved perceptual sensitivity, the latter of which distinguished BD from SZ. The relationship between gaze perception and broader socio-emotional functioning in SZ and $\mathrm{HC}$ was absent in $\mathrm{BD}$.

\section{KEYWORDS}

affective disorder, emotional intelligence, face processing, psychosis, schizophrenia, social cognition

\section{1 | INTRODUCTION}

Individuals with bipolar disorder (BD) often suffer from lasting functional impairments, even during remission of active mood symptoms. ${ }^{1-3}$ These impairments not only lead to reduced work productivity and unemployment, ${ }^{4}$ but also impact their social relationships and quality of life negatively. ${ }^{2,5}$ A main predictor of low functioning in $\mathrm{BD}$ is subsyndromal depressive symptoms (i.e., symptoms not meeting the full diagnostic criteria for a mood episode), ${ }^{2}$ but it only accounts for a small amount of variance ${ }^{6,7}$-even smaller (7\%) after accounting for 
the effect of neurocognitive functioning. ${ }^{8}$ Neurocognition is a more promising predictor, although the variance explained is typically in the range of $7 \%-21 \% .{ }^{8,9}$ Research with individuals diagnosed with schizophrenia (SZ) has shown that social cognition mediates the relationship between neurocognition and functional outcome, ${ }^{10,11}$ suggesting that social cognition has a more direct relationship to functioning. There is preliminary evidence showing a significant link between the two in BD. ${ }^{12-14}$ Given its potential as a better predictor of psychosocial outcome than neurocognition, a better understanding of social cognition in BD could inform the illness mechanism and refine current treatment.

Despite its clinical relevance, social cognition in BD remains underinvestigated, and findings are mixed. There is some evidence that individuals with $\mathrm{BD}$ are impaired in emotion processing (i.e., the ability to recognize, appraise, and utilize emotion) and theory of mind (i.e., the ability to infer other people's mental states, such as beliefs, intentions, and emotions, based on available social cues and contexts). ${ }^{15,16}$ However, not all studies have found social cognition impairment in $B D$, even in the presence of impaired neurocognition. ${ }^{17}$ Clinical factors are one potential source of variability across studies. For example, social cognitive deficits were more severe in BD patients with a history of psychotic symptoms than in those without. ${ }^{18}$ They were most severe during manic episodes, ${ }^{16,19}$ but could persist during remission of active mood symptoms. ${ }^{16}$ Another source of variability is the tasks used to assess social cognition. Some data suggest that social cognitive impairment in BD may be present only in some specific domains (e.g., selfreferential information processing) ${ }^{20}$ Despite evidence for significant genetic and phenotypic overlap between SZ and BD, the degree to which social cognitive abilities differ across the two disorders has not been extensively investigated. Although one study has found that the level of impairment (e.g., in theory-of-mind tasks) in BD is as severe as in SZ patients, ${ }^{21}$ the majority have found it to be intermediate between levels of healthy controls $(\mathrm{HC})$ and SZ. ${ }^{13,15,22}$

To determine if and how social cognition is compromised in BD, it is necessary to examine well-defined social cognitive domains. One core building block of social cognition is eye gaze perception. ${ }^{23}$ Humans develop the ability to infer attention and intention of others from their gaze direction during infancy and this ability is critical to successful social development and functioning. ${ }^{24}$ Abnormal gaze perception may lead to wrong judgments about the focus of people's attention, thus assigning meaning to irrelevant stimuli. For example, misperceiving someone else as looking at you may lead to grandiose ideas (if the intention is perceived as positive) or paranoid delusions (if the intention is perceived as negative). Studies have shown that self-referential gaze perception is impaired in SZ. ${ }^{25-27}$ Specifically, when gaze perception was assessed using a continuum of gaze directions and analyzed using a psychophysical method, SZ patients over-perceived eye contact when gaze was ambiguous and began to endorse eye contact with a weaker eye-contact signal (i.e., when gaze direction was more averted) compared with $\mathrm{HC}^{27}$ Their perception curve, plotting eye-contact perception as a function of gaze direction, was also shallower, suggesting more uncertainty or reduced sensitivity in making self-referential vs non-self-referential judgments of eye gaze. This impairment was correlated with more severe clinical symptoms and explained a significant amount of variance in socio-emotional functioning even after controlling for basic neurocognition. The same linear relationship between gaze perception and socio-emotional functioning was also observed in $\mathrm{HC}$, suggesting that gaze perception may be a determinant of social functioning regardless of disease status.

This study addresses a gap in the field of social cognition research in $\mathrm{BD}$, namely, gaze perception. Demonstrating a relationship between gaze perception and broader social functioning, as observed in SZ and $\mathrm{HC}$, would inform the mechanisms and treatment of functional impairment of BD. This would also provide support that gaze perception is an important dimension of social functioning that cuts across disease boundaries, thus enhancing our understanding of psychopathologies. Further, given preliminary findings that BD with psychotic features had more severe social cognitive deficits than those without, ${ }^{18}$ investigating how a history of psychosis is associated with a specific social cognitive deficit in BD, eye gaze perception in this case, would also inform disease mechanisms.

The primary aim of this study was to characterize self-referential eye gaze perception in BD and examine its implications for psychosocial functioning. In addition, to investigate whether putative gaze perception differed quantitatively or qualitatively from that in SZ, we examined its relationship to socio-emotional functioning in BD in comparison with $\mathrm{HC}$ and SZ patients. We used a psychophysical approach to examine judgments of eye contact as a function of eye-contact signal strength (i.e., gaze direction) as described in our previous study. ${ }^{27}$ Briefly, we used a relatively large number of trials of face stimuli covering the full range of gaze directions (from averted to direct in gradual increments). This method allowed us to examine two critical characteristics of eye-contact perception: thresholds (how strong an eyecontact signal one needs to perceive gaze as self-directed) and slope (how categorical or sensitive one's eye-contact perception is). We also manipulated head orientation (forward or averted) and facial emotion (neutral or fearful) of the face stimuli, because these two factors have been shown to interact with gaze direction during gaze perception. ${ }^{28,29}$ Studying how these two factors modulate eye-contact perception in $\mathrm{BD}$ can provide a richer understanding of how contextual and affective information influences self-referential gaze processing in the disorder.

We hypothesized that (i) BD patients would show abnormal eyecontact perception (i.e., overperception when gaze is ambiguous, and reduced perception thresholds and slope) compared with HC; (ii) the patterns of abnormalities in eye gaze perception in BD would be similar to those observed in SZ but to a lesser degree; (iii) the abnormalities in eye gaze perception would be worse in $\mathrm{BD}$ patients with a history of psychosis compared with those without; and (iv) BD patients would exhibit a similar relationship between gaze perception and socioemotional functioning as in SZ and HC.

\section{2 | METHODS}

\section{1 | Participants}

The sample consisted of 113 participants: 30 diagnosed with BD (16 with a history of psychosis and 14 without), $37 \mathrm{HC}$, and 46 participants 
diagnosed with schizophrenia or schizoaffective disorder (SZ). Data for $23 \mathrm{HC}$ and $26 \mathrm{SZ}$ patients were reported in a previous study. ${ }^{27} \mathrm{DSM}-\mathrm{IV}$ diagnoses were established using the Structured Clinical Interview for the DSM-IV (SCID-IV) ${ }^{30}$ or the Diagnostic Interview for Genetic Studies (DIGS Version 4.0). ${ }^{31}$ Participants were recruited through advertisements and referrals by clinicians and researchers in an academic medical center. BD and SZ patients were excluded if they had a history of alcohol/substance use disorder in the past 6 months. HC were excluded if they had any past or current Axis I disorders, alcohol/ substance use disorder in the past 5 years, or a first-degree relative with a psychotic or bipolar disorder. All participants were able to give informed consent and had at least 20/30 visions according to a Snellen chart. Written informed consent was obtained from every participant after a complete description of the study. The study was approved by the University of Michigan Medical School Institutional Review Board.

\subsection{Assessments}

The revised version of the Beck Depression Inventory (BDI-IA) ${ }^{32}$ and the Young Mania Rating Scale (YMRS) ${ }^{33}$ were used to assess participants' mood state. The Scale for Assessment of Positive Symptoms (SAPS) ${ }^{34}$ and the Scale for Assessment of Negative Symptoms (SANS)

${ }^{35}$ were used to assess the positive and negative symptoms of patients, respectively. To allow a direct comparison with previous findings in SZ, the Brief Assessment of Cognition of Schizophrenia (BACS) ${ }^{36}$ and the Mayer-Salovey-Caruso Emotional Intelligence Test (MSCEIT) ${ }^{37}$ were used to assess participants' neurocognition and socio-emotional functioning, respectively. The BACS is a performance-based test battery that assesses verbal memory, working memory, motor speed, attention, executive functions, and verbal fluency. MSCEIT is a performance-based battery that measures individuals' ability to perceive, facilitate, understand, and manage emotions.

\section{3 | Eye-contact perception task}

Participants viewed black-and-white photos of faces varying in head orientation (forward, or $30^{\circ}$ averted to left or right), emotion (neutral or fearful), and eye-contact signal strength $(0,0.1,0.2, \ldots, 1.0)$. Gaze direction varied from averted ( 0 eye-contact signal strength) to direct (1.0 eye-contact signal strength) in ten 10\% increments (see Figure 1 for example stimuli). The task contains 528 trials in total: 2 head orientations $\times 2$ emotions $\times 11$ eye-contact signal strengths $\times 6$ actors $\times 2$ directions (left or right). For each face, participants were instructed to indicate, according to their first impression, whether they felt the person was looking at them (yes/no) by pressing one of two buttons. The task was self-paced and participants were allowed to pause and take a brief break whenever they needed (see Tso et al. ${ }^{27}$ for more task details). The task typically lasted 10-12 minutes.

\section{4 | Data processing}

The major analyses were conducted on the parameters that define each participant's psychometric curve, plotting eye-contact perception as a function of gaze direction. To this end, a two-parameter logistic function was fitted to each participant's eye-contact endorsement rate (percentage of "yes, looking at me" responses) plotted against eye-contact signal strength:

$$
f(x)=1 /\left(1+c \cdot b^{x}\right)
$$

where $c$ and $b$ are constant parameters provided by the IBM SPSS Statistics 22 (IBM Corp., Armonk, NY, USA) Curve Estimation (logistic) function (see Figure 2 for an example). Because responses to averted faces did not approach a logistic function, only responses to forward faces were used in this analysis. Two psychophysical properties of eyecontact perception were derived from the fitted curve: thresholds (i.e., the expected signal strength given a certain eye-contact endorsement rate) and slope. Our previous study ${ }^{27}$ suggests that thresholds estimated using lower response cut-offs could best distinguish patients with SZ from HC. In this study, we used the same method and obtained nine perception thresholds using response cut-offs of $10 \%, 20 \%, \ldots$, $90 \%$ eye-contact endorsement rate. The slope of the function when $f(x)=50 \%$ was used as a measure of participants' sensitivity to eyecontact signal strength, given that it measures how rapidly one's perception changes from non-self-referential to self-referential (see Tso

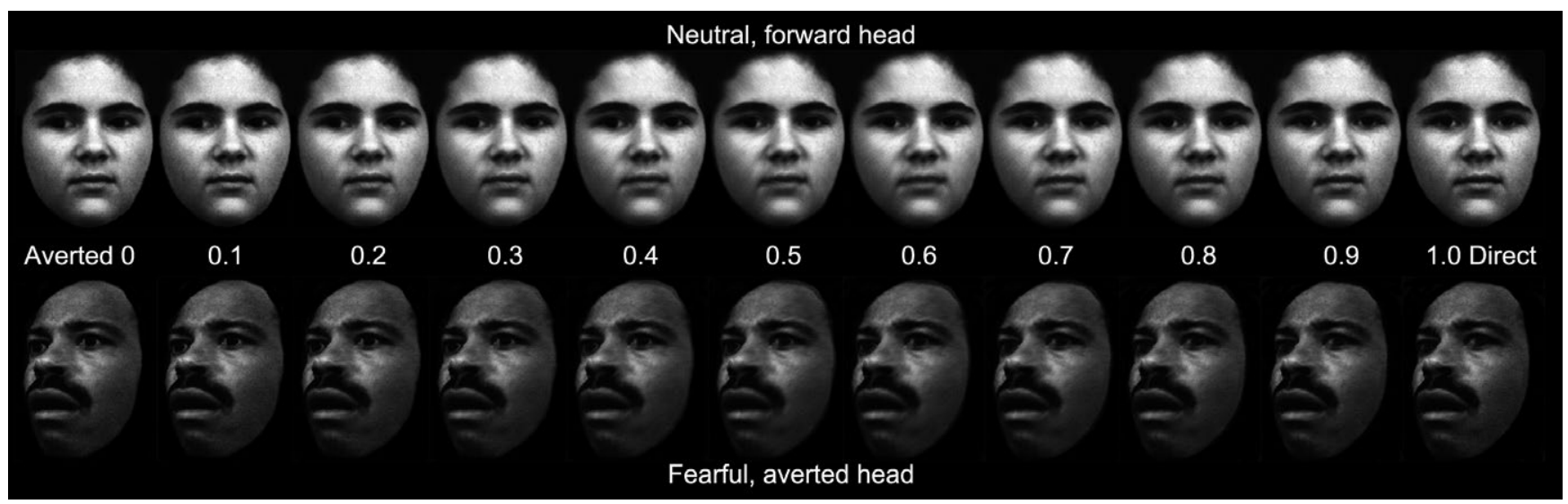

FIG URE 1 Sample face stimuli from the eye-contact perception task. From left to right, eye-contact signal strength increases in 10\% increments from 0 (averted) to 1.0 (direct) 


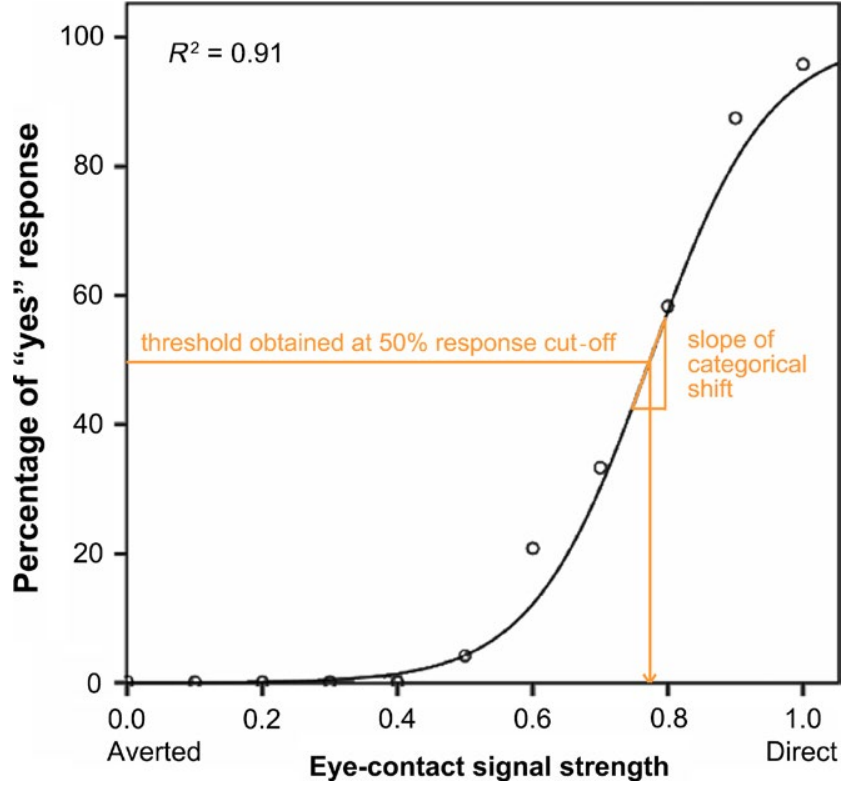

FIGURE 2 An example of a participant's psychometric curve, plotting eye-contact endorsement rate as a function of eye-contact signal strength. Nine perception thresholds were obtained using response cut-offs from $10 \%$ to $90 \%$ eye-contact endorsement rate. The slope of the function when endorsement rate $=50 \%$ was used as a measure of participants' sensitivity to eye-contact signal strength, i.e. the rate of change of the categorical shift from nonself-referential to self-referential [Colour figure can be viewed at wileyonlinelibrary.com]

et al. ${ }^{27}$ for more details on the mathematical derivation of the slope); higher values indicate that perception is more clear-cut categorical (i.e., with higher perceptual sensitivity).

In order to include the data on averted faces, we performed an additional analysis in which we calculated each subject's mean eyecontact endorsement rate for the 11 gaze angles.

\section{5 | Statistical analyses}

Group and model comparisons were conducted using Bayes factors (BFs) throughout this paper. The BF is the ratio of the Bayesian evidence of the numerator model (e.g., alternative hypothesis) to that of the denominator model (e.g., null hypothesis); it provides information regarding the relative strength of evidence of two competing models, instead of merely accepting/rejecting null hypotheses as in traditional frequentist statistics. Model complexity is penalized in the computation of model evidence, allowing a more parsimonious model to win if it fits the data better. $B F<1$ indicates evidence favoring the denominator model, while BF $>1$ indicates evidence favoring the numerator model. Interpretations of strength of evidence followed accepted guidelines, ${ }^{38}$ where a BF of 1-3 provides "anecdotal" evidence for the numerator model, 3-10 "substantial" evidence, 10-30 "strong" evidence, 30-100 "very strong" evidence, and >100 "decisive" evidence. Similarly, a BF of 0.33-1 provides "anecdotal" evidence for the denominator model (often the null hypothesis), 0.10-0.33 "substantial" evidence, 0.033-0.10 "strong" evidence, 0.01-0.033 "very strong" evidence, and <0.01 "decisive" evidence. All BFs were computed using the R package "BayesFactor". 39

For threshold, the anovaBF command was used to compare ANOVA models consisting of all possible permutations consisting of group, emotion, response cut-off, and their interaction terms as fixed effects. The model with the highest BF (compared against a denominator model consisting of only subject as a random factor) was chosen as the winning model and is reported in the Results. This was then followed up by pairwise group comparisons of threshold at each response cut-off using the ttestBF command.

For eye-contact endorsement rate, the anovaBF command was used to select the winning model among ANOVA models consisting of all possible permutations of group, head orientation, emotion, and their interaction terms as fixed effects.

For the slope of the perception curve, the anovaBF command was used to select the winning model among ANOVA models consisting of all possible permutations of group, emotion, and their interaction term as fixed effects. Follow-up pairwise group comparisons were conducted using the ttestBF command.

Finally, the relationships between eye-contact perception measures and clinical/functional measures in the three groups were examined using Pearson's correlations and multiple regressions. In the regression analyses, we used the slope of the gaze perception curve as a predictor, because it was the gaze perception measure that significantly explained socio-emotional functioning in SZ patients and $\mathrm{HC}$ in our previous study. ${ }^{27}$ This variable, in addition to group membership, was included as a predictor of MSCEIT. Specifically, the ImBF command was used to identify a winning model among regression models consisting of all possible permutations of group membership (coded as two dummy variables, SZ and BD, to denote the three groups), slope of the gaze perception curve, and their interaction terms as predictors of MSCEIT. Since HC was coded as the reference group, including the interaction terms (e.g., BD $\times$ gaze slope) allowed testing of whether the relationship between gaze slope and MSCEIT in one diagnostic group (e.g., BD patients) was different from that in $\mathrm{HC}$.

\section{3 | RESULTS}

\section{1 | Participant characteristics}

The BD, SZ, and HC groups were well matched for age and parental education. The BD group had a significantly lower male-to-female ratio than the other two groups. The BD group's socio-emotional functioning as measured by MSCEIT was not different from that of $\mathrm{HC}$, but the SZ group had a lower MSCEIT score than HC. See Table 1 for detailed participant characteristics.

\section{2 | Over-perception of eye contact}

For threshold, BFs of all possible ANOVA models showed that the winning model, providing "decisive" evidence ( $B F=1.26 \times 10^{662}$ ), contained group, emotion, response cut-off, group $\times$ emotion 
TABLE 1 Participant characteristics

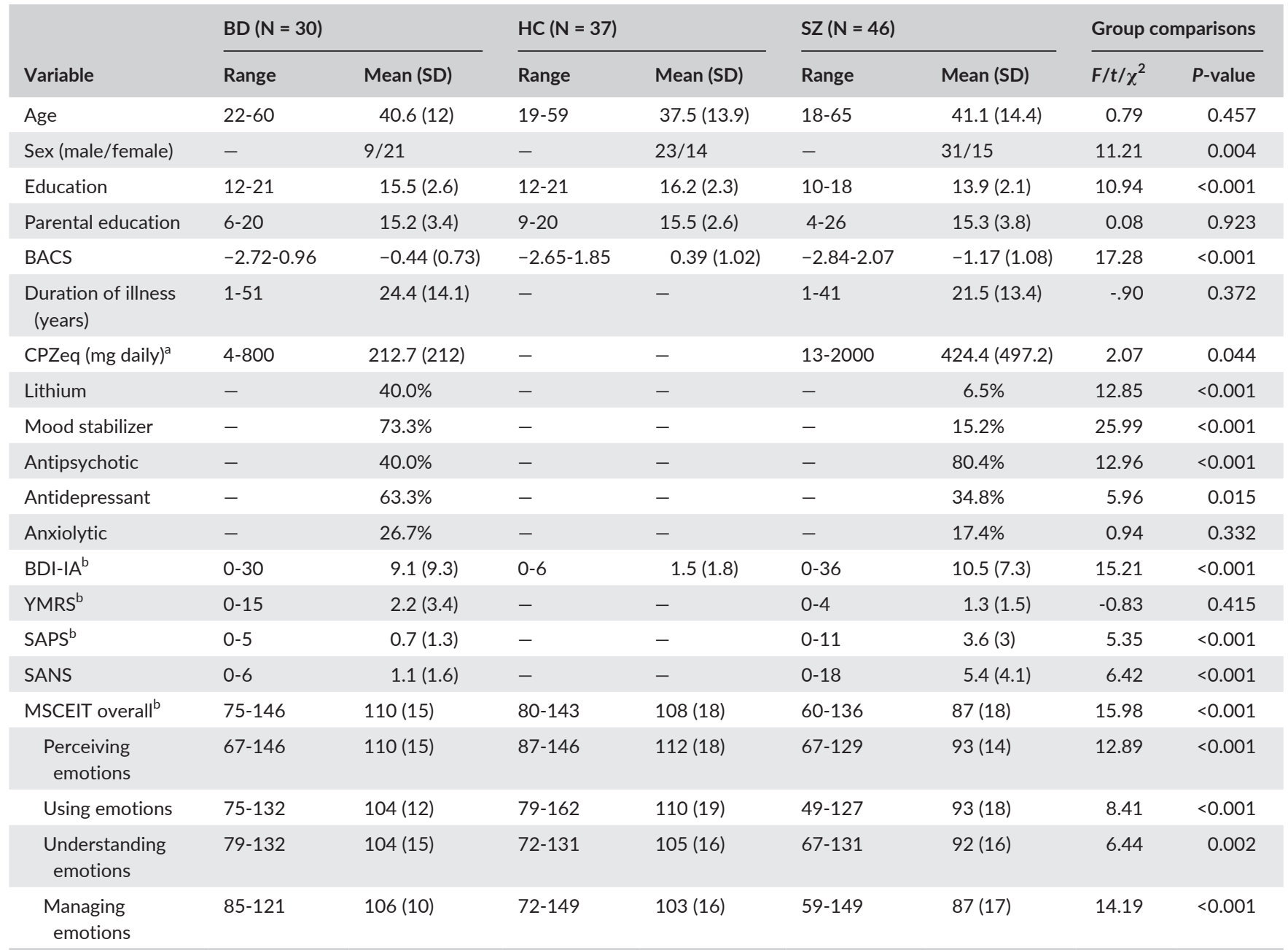

BACS, Brief Assessment of Cognition for Schizophrenia composite score; BD, individuals with bipolar disorder; BDI-IA, revised version of the Beck Depression Inventory; CPZeq, antipsychotic dose in chlorpromazine equivalent; HC, healthy controls; MSCEIT, age- and gender-adjusted scores on the Mayer-Salovey-Caruso Emotional Intelligence Test; SANS, Scale for the Assessment of Negative Symptoms; SAPS, Scale for the Assessment of Positive Symptoms; SZ, individuals with schizophrenia; YMRS, Young Mania Rating Scale.

${ }^{a}$ Analysis only included $37 \mathrm{SZ}$ and $12 \mathrm{BD}$ patients who were taking antipsychotics.

${ }^{b} 10$ SZ patients and 14 HC did not have data on SAPS, BDI-IA, YMRS, and MSCEIT because the data came from different studies.

interaction, and group $\times$ response cut-off interaction as fixed effects. The evidence for this model was more than 126 times stronger than that for the next best model (containing all factors except the group $\times$ emotion interaction). The group effect indicated that the overall threshold for the BD group (mean $=0.64$, standard deviation $[S D]=0.16$ ) was substantially lower than that for the $\mathrm{HC}$ group (mean $=0.74, \mathrm{SD}=0.14 ; \mathrm{BF}=3.89$ ) but did not differ from that for the $\mathrm{SZ}$ group (mean $=0.60, \mathrm{SD}=0.17 ; \mathrm{BF}=0.36$ ). The SZ group's overall threshold was "very strongly" lower than that of the HC group (BF = 90.18); that is, both BD and SZ patients needed weaker signal strength to indicate that eye gaze was directed towards them. The emotion effect indicated that, overall, the mean threshold for neutral faces (mean $=0.64, \mathrm{SD}=0.16$ ) was decisively lower than that for fearful ones $\left(\right.$ mean $\left.=0.69, \mathrm{SD}=0.20 ; \mathrm{BF}=6.19 \times 10^{5}\right)$. The group $\times$ emotion interaction was driven by a clear emotion effect in $\mathrm{BD}$ patients and $\mathrm{HC}$ (neutral < fearful; BF = 115 for BD and 266 for $\mathrm{HC}$ ) but only "anecdotal" evidence for it in SZ patients $(B F=2.50)$. Follow-up pairwise group comparisons at each response cut-off revealed what drove the group $\times$ response cut-off interaction. See Figure 3 for eyecontact perception thresholds of each group calculated using nine response cut-offs collapsed across the two emotions. Group differences increased as the response cut-off value to obtain the threshold decreased. This was true for both $\mathrm{BD}-\mathrm{HC}$ comparisons and SZ-HC comparisons. BD patients started to show clear evidence (BF > 3) of a lower threshold than $\mathrm{HC}$ at a response cut-off of $50 \%$ and the group difference reached its maximum at a response cut-off of $10 \%$. The pattern was the same for SZ patients, but the SZ < HC difference was even larger and started sooner at a response cut-off of $70 \%$. There were no differences in threshold between BD and SZ patients (BFs ranging from 0.24 to 0.66 ).

For eye-contact endorsement rate, results of BFs showed that the winning model contained fixed factors of group, signal strength, emotion, head orientation, group $\times$ signal strength, emotion $\times$ signal strength, group $\times$ head orientation, signal strength $\times$ head orientation, 


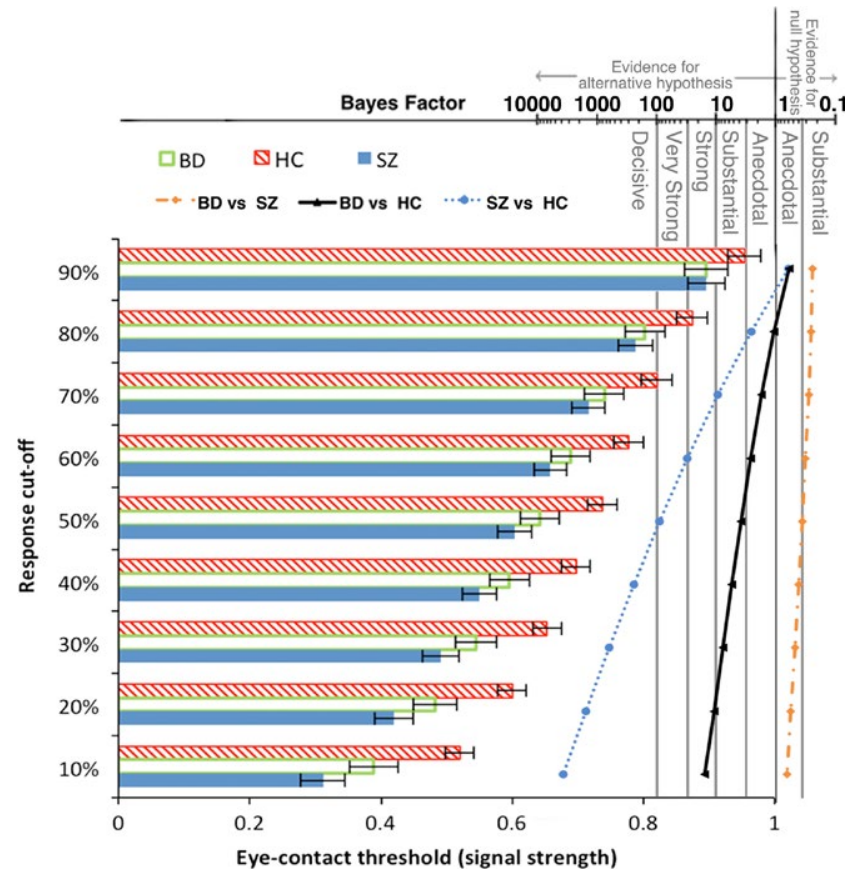

FIGURE 3 Group differences in eye-contact perception threshold (forward face only) increased as response cut-off (criterion used to obtain threshold) decreased. The corresponding Bayes factors (scale shown at the top of the figure) of pairwise group differences at each response cut-off value are plotted to the right of the bars [Colour figure can be viewed at wileyonlinelibrary.com]

emotion $\times$ head orientation, group $\times$ signal strength $\times$ head orientation, and emotion $\times$ signal strength $\times$ head orientation. The evidence of this model was overwhelmingly "decisive" ( $\mathrm{BF}=3.34 \times 10^{1616}$ ), which was more than 167 times stronger than the next best model (containing all but two of the fixed effects in the winning model: group $\times$ head orientation and group $\times$ signal strength $\times$ head orientation). Group patterns of eye-contact endorsement rate across eye-contact signal strengths and head orientations collapsed across the two emotions (because there was no interaction between group and emotion) are presented in Figure 4. Overall, BD patients (mean $=30 \%, S D=14 \%$ ) endorsed eye contact more frequently than $\mathrm{HC}$ (mean $=23 \%, \mathrm{SD}=10 \%$; $\mathrm{BF}=3.60$ ), but did not differ from SZ patients (mean $=33 \%, S D=13 \% ; B F=0.33$ ). SZ patients endorsed eye contact very strongly more frequently than $\mathrm{HC}(\mathrm{BF}=98.91)$. The emotion effect indicated that the endorsement rate for neutral faces (mean $=31 \%, S D=13 \%$ ) for neutral faces was higher than that for fearful ones (mean $=26 \%, S D=13 \%$; $\left.\mathrm{BF}=1.19 \times 10^{24}\right)$. The head orientation effect indicated that the endorsement rate for forward faces (mean $=37 \%, S D=13 \%$ ) was higher than that for averted faces ( mean $=21 \%, S D=16 \% ; B F=8.02 \times 10^{23}$ ). The group $\times$ head orientation interaction was driven by a higher endorsement rate in $\mathrm{BD}$ patients ( mean $=37 \%, \mathrm{SD}=13 \%$ ) compared with $\mathrm{HC}$ (mean $=30 \%, \mathrm{SD}=10 \% ; \mathrm{BF}=3.59$ ), which was not different from that in SZ patients (mean $=41 \%, S D=13 \%$; BF $=0.42$ ) for forward faces. For averted faces, however, there was only "anecdotal" evidence that mean endorsement rate in BD patients (mean $=23 \%, \mathrm{SD}=17 \%$ ) was higher than that in $\mathrm{HC}$ (mean $=15 \%, \mathrm{SD}=11 \%$; $\mathrm{BF}=1.88$ ) but substantial evidence that it did not differ from that in SZ (mean $=25 \%$, $S D=16 \% ; B F=0.27)$. As for the group $\times$ signal strength interaction, follow-up pairwise group comparisons at each signal strength revealed that group differences were the largest in the low to middle range of signal strengths for both BD-HC comparisons and SZ-HC comparisons. There was no difference in mean endorsement rate between the $\mathrm{BD}$ and $\mathrm{SZ}$ groups across signal strengths.

\section{3 | Intact perceptual sensitivity in BD}

The gaze perception slope measures how rapidly one's perception changes from non-self-referential to self-referential, and is thus an index of perceptual sensitivity. For the slope, results for BFs showed that the winning model, providing "very strong" evidence ( $B F=87.98)$, contained main effects of group and emotion only, with no group $\times$ emotion interaction (see Figure S1 for BFs of all possible models). Follow-up analyses of the group effect showed that there was no evidence for a difference in gaze perception slope between $\mathrm{BD}$ patients (mean $=2.39, \mathrm{SD}=0.67$ ) and $\mathrm{HC}$ (mean $=2.60$, $\mathrm{SD}=0.44$; Cohen's $d=0.37, \mathrm{BF}=0.69$ ), or between BD and SZ patients (mean $=2.11, \mathrm{SD}=0.64 ; d=0.43, \mathrm{BF}=0.99$ ). The evidence for a shallower slope in SZ patients relative to $\mathrm{HC}$ was "decisive" ( $d=0.89$, $B F=140.49)$. The emotion effect indicated that the slope for neutral faces $($ mean $=2.67, \mathrm{SD}=0.72$ ) was much steeper than that for fearful faces (mean $=2.50, \mathrm{SD}=0.66 ; \mathrm{BF}=21.21$ ) across participants

We conducted two follow-up BF analyses to further examine if gaze perception slope was different between subgroups within the BD group. To address the question of whether psychosis is a determinant of BD patients' gaze perception, we compared BD patients with and without a history of psychosis. The BF results favored no group difference in gaze perception slope $(B F=0.35)$, and the effect size of group difference was also small $(d=0.14)$. Because the BD group had a higher female:male ratio relative to the $\mathrm{SZ}$ and $\mathrm{HC}$ groups and the literature suggests that female individuals generally have better social cognition than male individuals, ${ }^{40,41}$ we also examined whether there were differences in gaze perception slope as well as MSCEIT between female and male BD participants. BF results favored a lack of sex difference in the slope of gaze perception ( $B F=0.47 ; d=0.04$ ) as well as MSCEIT (BF $=0.37 ; d=0.02$ ).

\subsection{Relationship with clinical and functional measures}

In both patient groups, eye-contact perception was not significantly correlated with mood symptoms (BDI-IA and YMRS), positive symptoms (SAPS), or negative symptoms (SANS; see Table S1 for pairwise correlations). In the BD and $\mathrm{HC}$ groups, eye-contact perception was not significantly correlated with neurocognition (BACS). However, in SZ patients, altered eye-contact perception (lower perception threshold at low/medium response cut-offs; reduced slope of categorical shift) was significantly correlated with poorer neurocognition.

To examine the relationship between gaze perception and socioemotional functioning (MSCEIT) in each group, we assessed the BFs of 


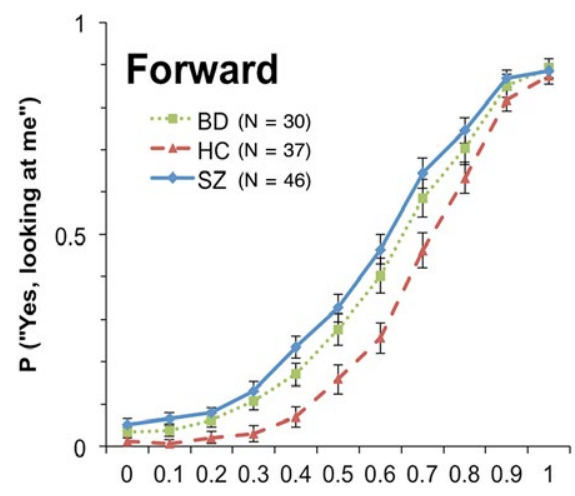

Eye-contact signal strength

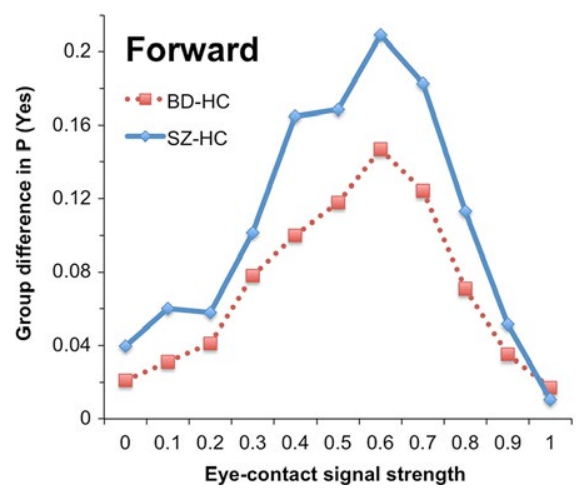

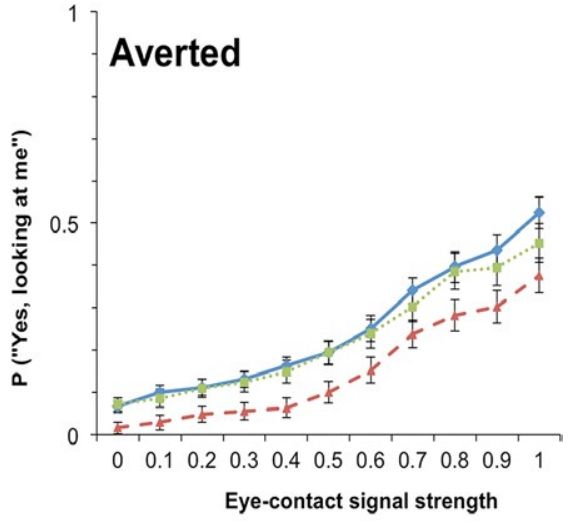

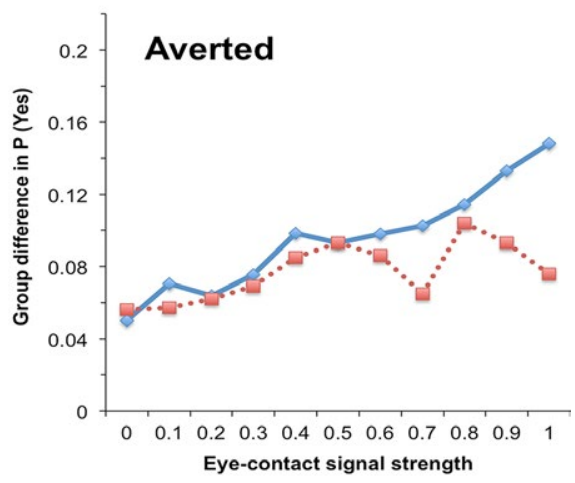

FIGURE 4 Upper panels: eye-contact endorsement rate (percentage of "yes, looking at me" responses) by group along the gaze continuum. P, percentage. Lower panels: over-perception of eye contact of patients with bipolar disorder (BD) and schizophrenia (SZ) along the gaze continuum (i.e., group differences between BD patients and healthy controls $[\mathrm{HC}]$, and between SZ patients and $\mathrm{HC}$ ) [Colour figure can be viewed at wileyonlinelibrary. com] different linear regression models. The model with the most "decisive" evidence $\left(B F=1.84 \times 10^{7}\right)$ contained gaze perception slope, membership of the SZ group, membership of the BD group, and the interaction term between slope and BD group membership as predictors of MSCEIT; membership of the HC group was the implicit reference group. The evidence of this model was nearly nine times stronger than that of the next best model that did not include the interaction term (see Figure S2 for BFs of all models). This winning model suggested that: (i) a steeper gaze perception slope was associated with better MSCEIT; (ii) the three groups had different intercepts, i.e., different MSCEIT scores when the slope was held at zero; and (iii) the BD (but not SZ) group had a different (reduced) linear relationship between gaze perception slope and MSCEIT relative to $\mathrm{HC}$-indicating that gaze perception slope had less effect on MSCEIT in the BD group compared with $\mathrm{HC}$ (see Figure 5). The regression model explained $45.6 \%$ of variance in MSCEIT ( $F=16.6, P<0.001)$.

\section{4 | DISCUSSION}

This study examined whether BD patients showed abnormal selfreferential gaze perception-a crucial perceptual component of
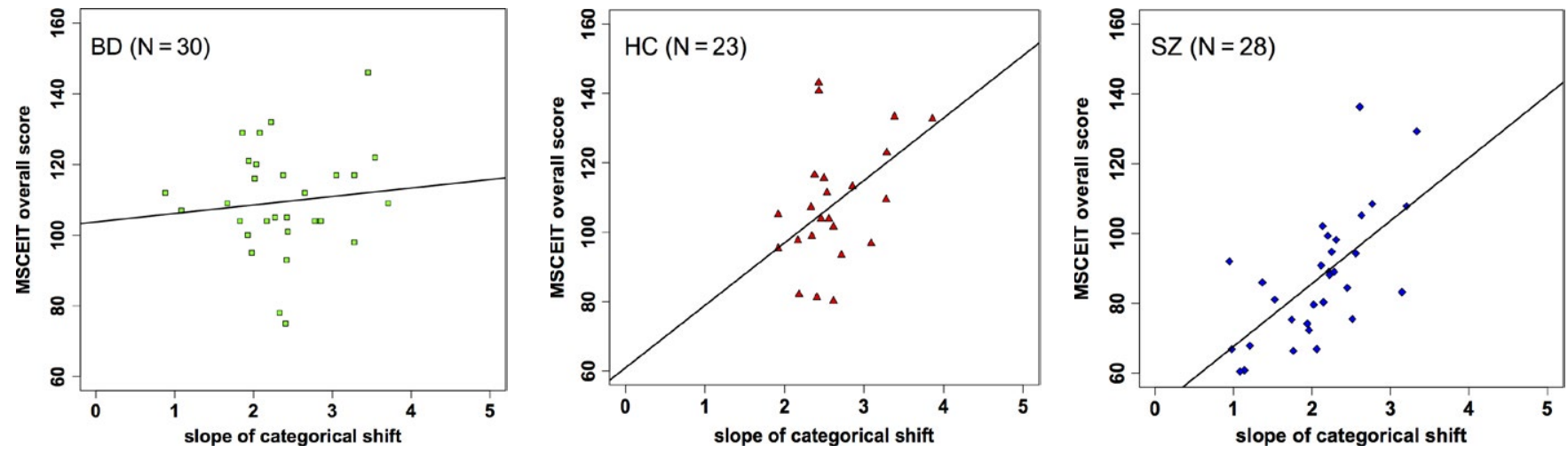

FIGURE 5 Scatterplots of Mayer-Salovey-Caruso Emotional Intelligence Test (MSCEIT) score against slope of categorical shift with the regression line of the best model among bipolar disorder (BD) patients (left), healthy controls (HC) (middle), and schizophrenia (SZ) patients (right). A faster rate at which perception changes from non-self-referential to self-referential directly correlates with better social cognitive performance in $\mathrm{HC}$ and SZ patients [Colour figure can be viewed at wileyonlinelibrary.com] 
complex social processes-and whether putative abnormality was related to socio-emotional functioning. Using a psychophysics approach, we found that on some measures of eye-contact perception, BD patients were indistinguishable from SZ patients. Like SZ patients, $\mathrm{BD}$ patients were more likely than $\mathrm{HC}$ to report a face to be making eye contact with them, particularly as gaze direction became increasingly averted. BD patients required a weaker eye-contact signal (i.e., less direct gaze angle) to start perceiving gaze as self-directed, relative to HC. Unlike SZ patients, however, the slope of the gaze perception curve of BD patients was not different from that of $\mathrm{HC}$, indicating that, as actual gaze became increasingly directed at the participant, gaze perception in BD patients changed from non-self-referential to self-referential as rapidly as in $\mathrm{HC}$; that change simply occurred sooner (i.e., at a weaker eye-contact signal strength) in $\mathrm{BD}$ patients than in HC. Taken together, our findings suggest that BD patients overperceive eye contact from a gaze that $\mathrm{HC}$ would consider ambiguous or non-self-directed, but their perceptual sensitivity was preserved.

We hypothesized that BD patients would exhibit a similar relationship between gaze perception (slope) and socio-emotional functioning (MSCEIT) as in SZ patients and HC. However, while higher gaze perception slope was associated with better MSCEIT score in SZ patients and $\mathrm{HC}$, this relationship was absent in BD patients. It is noteworthy that, although BD patients showed a self-referential bias in gaze perception, their MSCEIT scores did not differ from those of HC, consistent with previous reports. ${ }^{42,43}$ Since reasoning and problem-solving skills appear to be intact in $\mathrm{BD},{ }^{42}$ it is possible that this compensates for altered perception of social signals, leaving socio-emotional functions, at least as measured by MSCEIT, unaffected. This highlights the issue that social cognitive instruments well validated in SZ may not have the same utility in BD despite the clinical and cognitive overlap between the two disorders.

Another way in which performance differed between BD and SZ patients was in the extent to which self-referential biases in gaze perception were influenced by emotion. Both $\mathrm{BD}$ patients and $\mathrm{HC}$ required more direct eye gaze to endorse the person as looking at them when the face was fearful than when it was neutral. This effect of facial emotion was blunted in SZ patients. Such emotion effect on gaze perception is consistent with previous findings in healthy individuals that fearful faces bias people to perceive averted gaze, which could be a result of fearful emotion and averted gaze being congruent in avoidant motivation. ${ }^{29,44}$ Therefore, it seems that, despite an overall tendency for over-perceiving gaze as self-referential, BD patients showed normal emotional modulation of gaze perception, which distinguished them from SZ patients. This preserved integration of affective contexts in self-referential social signal processing may be a contributing factor of normal socio-emotional functioning in this BD sample.

The findings of differential gaze perception between BD and SZ groups in this study have important treatment implications. They suggest that gaze perception deficits in BD and SZ may be driven by different factors, which may have differential relationships with socio-emotional functioning. Many social cognitive trainings have been developed for $\mathrm{SZ}^{45}$ and there has been effort to apply such interventions to $\mathrm{BD},{ }^{46}$ assuming that, if improvement in those specific social cognitive domains results in improved functional outcome in $\mathrm{SZ}$, the same would happen in BD. Our findings suggest that this may not necessarily be the case. For example, BD participants showed a self-referential bias but preserved perceptual sensitivity, suggesting that abnormal gaze perception in BD likely reflects a top-down problem rather than impaired data-driven perception as observed in SZ. Therefore, interventions for BD should focus on top-down processes (e.g., cognitive restructuring as implemented in cognitive-behavioral therapy; brain stimulation targeting frontal regions); cognitive training paradigms that aim to strengthen early sensory processing for SZ 47 may not be as beneficial. The differential relationship between gaze perception and socio-emotional functioning in $\mathrm{BD}$ and $\mathrm{SZ}$ suggests that BD and SZ patients might make social inferences using different strategies or sources of information. Future investigations of the cognitive and neural bases of these strategies would enhance our understanding of differences in social cognition and treatment response between psychiatric disorders as well as across individuals, helping to develop more informative assessment and personalized treatment.

We did not find any significant correlations between gaze perception and positive/negative symptoms in the patient groups. We also did not find any significant differences in gaze perception between $\mathrm{BD}$ patients with and without a history of psychosis, suggesting that abnormal self-referential gaze perception may not be a marker of psychotic symptoms. This is consistent with several previous studies showing that BD patients with and without psychotic symptoms displayed no difference in their performances of various theory-of-mind tasks, ${ }^{48-50}$ although one study has reported that BD patients with psychosis performed worse than those without in the perceptual and reasoning aspects of social cognition, measured with tasks of emotion recognition and logical arrangement of pictures depicting social scenarios. ${ }^{18}$ It is also possible that altered gaze perception is only related to specific aspects of psychosis such as paranoia, and therefore correlating it with SAPS total score may not be able to capture a true relationship. Given that our analysis was limited by a modest sample size and that previous inconsistent findings in this area may be due to methodological differences, larger studies with more comprehensive assessment of social cognition are needed to more conclusively show whether a history of psychosis in BD has any impact on gaze perception and its relationship to social cognition in general. Further, since most patients in this study were clinically stable and euthymic, it is possible that the null result of symptom correlates of gaze perception in BD was due to the limited range of symptom severity in both groups, as was the case in other studies. ${ }^{17}$ It remains to be investigated whether impaired gaze perception varies between mood phases in BD.

The interpretation of the current findings is limited by several factors. First, the BD group had a higher female:male ratio relative to the other two groups. Previous social cognition studies in healthy individuals found that women perform better than men. ${ }^{40,41} \mathrm{~A}$ recent meta-analysis of social cognitive studies comparing BD and SZ patients also showed that larger effect sizes (BD patients better than $\mathrm{SZ}$ patients) were associated with higher male-to-female ratios in the SZ group. ${ }^{22}$ Although we conducted additional analyses to rule out the possibility that the intact gaze perception slope and MSCEIT 
performance in our BD sample were due to better performance of the female BD participants, the sample size of male BD patients in this study was undeniably small and it is difficult to draw definite conclusions on potential sex differences in social cognition in BD. This question needs to be addressed in future studies with larger samples that have balanced female:male ratios. Second, different medication regimens could be a potential confound. Our sample size did not permit analysis of subgroups of BD and SZ patients who were on similar medication regimens. Because the use and dose of medications are not independent of symptoms and are also confounded by individual treatment response, the question of whether medications contribute to the observed gaze perception abnormalities can only be adequately addressed by studying medication-free participants or those in the early stage of the illness in future studies. Third, the task comprised many (528) trials and there was a possibility that any poor performance may have been due to general cognitive deficits. Since frequent attentional lapses or random responses would result in "noisier" eyecontact endorsement rates for clearly averted and clearly direct gaze (i.e., significantly shifted away from $0 \%$ and $100 \%$ at both ends of the $x$-axis), response patterns of all three groups (see Figure 4) show that this was not the case. Although a self-referential bias in BD could be due to low-level perceptual deficits or general cognitive dysfunctions, BD patients were as sensitive to gaze signal strength as $\mathrm{HC}$ (as indicated by their equal perceptual slopes), making deficits in low-level perception seem unlikely. There was also no significant correlation between neurocognition (BACS) and gaze perception in BD patients, further ruling out general cognitive deficits as a contributor to their bias. The relationship between general cognitive functioning and gaze perception in SZ has been examined in detail in our previous study, and the findings showed that abnormal gaze perception in SZ is above and beyond just general cognitive deficits. ${ }^{27}$ Lastly, static visual stimuli were used in this study. There is evidence that different brain systems may be involved in processing static vs dynamic faces, and dynamic facial expressions may be more ecologically valid than static photos in emotion recognition studies. ${ }^{51}$ It remains to be investigated whether basic-level social cognition such as eye gaze perception is similarly affected by motion information.

\section{5 | CONCLUSIONS}

This study used a novel psychophysics approach to examine a fundamental social cognitive function, eye-contact perception, in BD. Our results showed that BD patients exhibited a similar selfreferential bias in eye gaze perception as SZ patients, characterized by over-perception of self-directed intention when viewing ambiguous gaze direction, and required weaker eye-contact signal strength to start perceiving eye contact. However, BD patients' categorical gaze perception was as efficient as HC's and their socio-emotional functioning did not appear to depend on gaze perception performance as those of $\mathrm{HC}$ and SZ patients do. These findings suggest that preserved perceptual sensitivity in making eye-contact judgment distinguishes BD from SZ patients and may suggest differential psychopathological mechanisms of social dysfunction between BD and SZ.

\section{ACKNOWLEDGEMENTS}

The authors thank Ms. Gloria Harrington for her assistance in subject recruitment and the participants for their participation in this study. This research was partially supported by the National Institutes of Health (5KL2TR000434 to I.F.T.), the National Institute of Mental Health (5K23MH108823 to I.F.T.; R21MH101676 to S.F.T.; R01MH082784 to V.E.R.), a NARSAD Young Investigator award from the Brain and Behavior Research Foundation (to K.T.), and the Heinz C. Prechter Bipolar Research Fund (a grant to M.G.M.).

\section{ORCID}

Beier Yao iD http://orcid.org/0000-0002-4842-5206

Ivy F Tso (iD http://orcid.org/0000-0002-0984-1186

\section{REFERENCES}

1. Grande I, Berk M, Birmaher B, Vieta E. Bipolar disorder. Lancet. 2016;387:1561-1572.

2. Sanchez-Moreno J, Martinez-Aran A, Tabarés-Seisdedos R, et al. Functioning and disability in bipolar disorder: an extensive review. Psychother Psychosom. 2009;78:285-297.

3. Saarni SI, Viertio S, Perälä J, et al. Quality of life of people with schizophrenia, bipolar disorder and other psychotic disorders. Br J Psychiatry. 2010;197:386-394.

4. World Health Organization. The global burden of disease: 2004 update. http://www.who.int/healthinfo/global_burden_disease/GBD_ report_2004update_full.pdf. Accessed April 6, 2017.

5. Dean BB, Gerner D, Gerner RH. A systematic review evaluating healthrelated quality of life, work impairment, and healthcare costs and utilization in bipolar disorder. Curr Med Res Opin. 2004;20:139-154.

6. Özer S, Uluşahin A, Batur S, et al. Outcome measures of interepisode bipolar patients in a Turkish sample. Soc Psychiatry Psychiatr Epidemiol. 2002;37:31-37.

7. Fagiolini A, Kupfer DJ, Masalehdan A, et al. Functional impairment in the remission phase of bipolar disorder. Bipolar Disord. 2005;7:281-285.

8. Martinez-Aran A, Vieta E, Torrent C, et al. Functional outcome in bipolar disorder: the role of clinical and cognitive factors. Bipolar Disord. 2007;9:103-113.

9. Tabarés-Seisdedos R, Balanzá-Martínez V, Sánchez-Moreno J, et al. Neurocognitive and clinical predictors of functional outcome in patients with schizophrenia and bipolar I disorder at one-year follow-up. J Affect Disord. 2008;109:286-299.

10. Couture SM, Penn DL, Roberts DL. The functional significance of social cognition in schizophrenia: a review. Schizophr Bull. 2006;32:S44-S63.

11. Hoe M, Nakagami E, Green MF, Brekke JS. The causal relationships between neurocognition, social cognition and functional outcome over time in schizophrenia: a latent difference score approach. Psychol Med. 2012;42:2287-2299.

12. Van Rheenen TE, Rossell SL. Phenomenological predictors of psychosocial function in bipolar disorder: is there evidence that social cognitive and emotion regulation abnormalities contribute? Aust New Zeal J Psychiatry. 2014;48:26-35.

13. Caletti E, Paoli RA, Fiorentini A, et al. Neuropsychology, social cognition and global functioning among bipolar, schizophrenic patients and healthy controls: preliminary data. Front Hum Neurosci. 2013;7:661. 
14. Tabak NT, Green MF, Wynn JK, et al. Perceived emotional intelligence is impaired and associated with poor community functioning in schizophrenia and bipolar disorder. Schizophr Res. 2015;162:189-195.

15. Samamé C. Social cognition throughout the three phases of bipolar disorder: a state-of-the-art overview. Psychiatry Res. 2013;210:1275-1286.

16. Bora E, Bartholomeusz C, Pantelis C. Meta-analysis of Theory of Mind (ToM) impairment in bipolar disorder. Psychol Med. 2016;46:253-264.

17. Lee J, Altshuler L, Glahn DC, et al. Social and nonsocial cognition in bipolar disorder and schizophrenia: relative levels of impairment. Am J Psychiatry. 2013;170:334-341.

18. Thaler NS, Allen DN, Sutton GP, et al. Differential impairment of social cognition factors in bipolar disorder with and without psychotic features and schizophrenia. J Psychiatr Res. 2013;47:2004-2010.

19. Hawken ER, Harkness KL, Lazowski LK, et al. The manic phase of bipolar disorder significantly impairs theory of mind decoding. Psychiatry Res. 2016;239:275-280

20. Zhao Y, Luo W, Chen J, et al. Behavioral and neural correlates of self-referential processing deficits in bipolar disorder. Sci Rep. 2016;6:24075

21. Donohoe G, Duignan A, Hargreaves A, et al. Social cognition in bipolar disorder versus schizophrenia: comparability in mental state decoding deficits. Bipolar Disord. 2012;14:743-748.

22. Bora $\mathrm{E}$, Pantelis $\mathrm{C}$. Social cognition in schizophrenia in comparison to bipolar disorder: a meta-analysis. Schizophr Res. 2016;175:72-78.

23. Itier RJ, Batty M. Neural bases of eye and gaze processing: the core of social cognition. Neurosci Biobehav Rev. 2009;33:843-863.

24. Butterworth $\mathrm{G}$. The ontogeny and phylogeny of joint visual attention. In: Whiten A, ed. Natural Theories of Mind: Evolution, Development and Simulation of Everyday Mindreading. Oxford: Basil Blackwell; 1991:223-232

25. Hooker C, Park S. You must be looking at me: the nature of gaze perception in schizophrenia patients. Cogn Neuropsychiatry. 2005;10:327-345.

26. Rosse RB, Kendrick K, Wyatt RJ, et al. Gaze discrimination in patients with schizophrenia: preliminary report. Am J Psychiatry. 1994:151:919-921.

27. Tso IF, Mui ML, Taylor SF, Deldin PJ. Eye-contact perception in schizophrenia: relationship with symptoms and socioemotional functioning. J Abnorm Psychol. 2012;121:616-627.

28. Itier RJ, Alain C, Kovacevic N, McIntosh AR. Explicit versus implicit gaze processing assessed by ERPs. Brain Res. 2007;1177:79-89.

29. Tipples J. Fear and fearfulness potentiate automatic orienting to eye gaze. Cogn Emot. 2006;20:309-320.

30. First MB, Spitzer RL, Gibbon M, Williams JB. Structured Clinical Interview for DSM-IV Axis I Disorders, Patient Edition. (SCID-P), version 2.0. New York, NY: Biometrics Research; 1995.

31. Nurnberger J, Blehar MC, Kaufmann CA, et al. Diagnostic interview for genetic studies. rationale, unique features, and training. Arch Gen Psychiatry. 1994;51:849-859.

32. Beck AT, Steer RA. Manual for the Beck Depresion Inventory. San Antonio, TX: Psychological Corporation; 1993.

33. Young RC, Biggs JT, Ziegler VE, Meyer DA. A rating scale for mania: reliability, validity and sensitivity. Br J Psychiatry. 1978;133:429-435.

34. Andreasen NC. Scale for the Assessment of Positive Symptoms (SAPS). lowa City, IA: University of lowa; 1984

35. Andreasen NC. Scale for the Assessment of Negative Symptoms (SANS). lowa City, IA: University of lowa; 1984
36. Keefe R, Goldberg TE, Harvey PD, et al. The brief assessment of cognition in schizophrenia: reliability, sensitivity, and comparison with a standard neurocognitive battery. Schizophr Res. 2004;68:283-297.

37. Mayer JD, Salovey P, Caruso DR. Mayer-Salovey-Caruso Emotional Intelligence Test. North Tonawanda, NY: Multi-Health Systems Inc.; 1999.

38. Jeffreys H. Theory of Probability, 3rd edn. Oxford, UK: Oxford University Press; 1961.

39. Morey RD, Rouder JN. BayesFactor: computation of bayes factors for common designs. R package version 0.9.12-2. 2015.

40. Stevens JS, Hamann S. Sex differences in brain activation to emotional stimuli: a meta-analysis of neuroimaging studies. Neuropsychologia. 2012;50:1578-1593.

41. Frank CK, Baron-Cohen S, Ganzel BL. Sex differences in the neural basis of false-belief and pragmatic language comprehension. Neurolmage. 2015;105:300-311.

42. Van Rheenen TE, Rossell SL. An empirical evaluation of the MATRICS consensus cognitive battery in bipolar disorder. Bipolar Disord. 2014;16:318-325.

43. Burdick KE, Goldberg TE, Cornblatt BA, et al. The MATRICS consensus cognitive battery in patients with bipolar I disorder. Neuropsychopharmacology. 2011;36:1587-1592.

44. Adams RB, Franklin RG. Influence of emotional expression on the processing of gaze direction. Motiv Emot. 2009;33:106-112.

45. Fiszdon JM, Reddy LF. Review of social cognitive treatments for psychosis. Clin Psychol Rev. 2012;32:724-740.

46. Lahera G, Benito A, Montes JM, et al. Social cognition and interaction training (SCIT) for outpatients with bipolar disorder. J Affect Disord. 2013;146:132-136.

47. Nahum M, Fisher M, Loewy R, et al. A novel, online social cognitive training program for young adults with schizophrenia: a pilot study. Schizophr Res Cogn. 2014;1:e11-e19.

48. Lahera G, Montes JM, Benito A, et al. Theory of mind deficit in bipolar disorder: is it related to aprevious history of psychotic symptoms? Psychiatry Res. 2008;161:309-317.

49. Benito A, Lahera G, Herrera S, et al. Deficits in recognition, identification, and discrimination of facial emotions in patients with bipolar disorder. Rev Bras Psiquiatr. 2013;35:435-438.

50. Martino DJ, Strejilevich SA, Fassi G, et al. Theory of mind and facial emotion recognition in euthymic bipolar I and bipolar II disorders. Psychiatry Res. 2011;189:379-384.

51. Alves NT. Recognition of static and dynamic facial expressions: a study review. Estud Psicol. 2013;18:125-130.

\section{SUPPORTING INFORMATION}

Additional Supporting Information may be found online in the supporting information tab for this article.

How to cite this article: Yao B, Mueller SA, Grove TB, et al. Eye gaze perception in bipolar disorder: Self-referential bias but intact perceptual sensitivity. Bipolar Disord. 2018;20:6069. https://doi.org/10.1111/bdi.12564 